\title{
$\mathbf{M}_{\mathrm{V}}-\sigma$ RELATION: A UNIVERSAL LAW?
}

\section{S.R. ZAGGIA}

Dip. di Astronomia, Univ. di Padova, Italy

Globular clusters (GC) of different galaxies are thought to be similar in their intrinsic nature, despite the very different environments in which they reside. This statement is mainly based on the luminosity function of GC that appear to be similar for galaxies structurally very different (Secker and Harris 1993). To support the above conclusion only few other GC parameters have been used: integrated colors and metallicity index (Harris 1991). Now, there is a growing body of GC velocity dispersions of other local group galaxies which can be used to perform a useful comparison. The tool for such a comparison is the correlation between GC total luminosity, $\mathrm{MV}_{\mathrm{V}}$, and velocity dispersion, $\sigma$, found by Djorgovski (1991). This is one of the best non trivial correlation of GC structural parameters that can give important answers to the problems of the formation and evolution of GCs.

To perform the comparison between local group GCs, we started from the velocity dispersions database for 56 Milky Way GCs (MW) of Pryor and Meylan (1993). It was updated to a total of $60 \mathrm{GCs}$, adding measures of $\sigma$ coming from our survey of velocity dispersion cusps in GCs (Zaggia et al. 1995), and some recently published measures (Peterson et al. 1994, 1995). Then, we added the data of Dubath et al. (1993a) for 10 old LMC clusters and for NGC 121, the only known old GC in the SMC (Stryker et al. 1985). Magellanic Cloud cluster total luminosities are from van den Bergh (1981). Finally, $\sigma$ and $M_{\mathrm{V}}$ for 3 Fornax GCs were measured by Dubath et al. (1993b). For M31 we used the only existing published set of 12 GCs velocity dispersions (Peterson 1989). We selected only the clusters with reliable $\sigma$ according to Peterson (1989), using for two of them (Bo 193, 225) an updated velocity dispersion given by Peterson (1993).

The total of 86 GCs in local group galaxies are plotted in Fig. 1: it is impressive to see that all the clusters follow the same correlation. The continuous line is drawn from the fit to the MW GCs sample, while the dashed line is the fit to the not-MW clusters: the two fits are almost iden- 


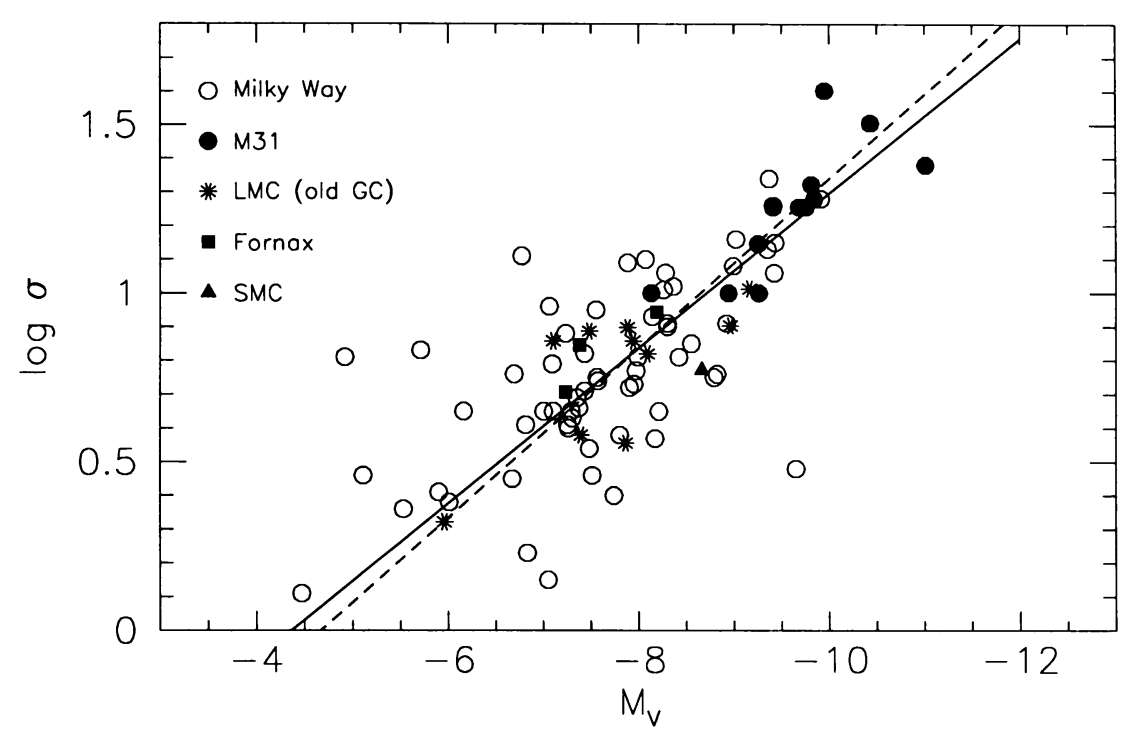

Figure 1. GC from different Local Group galaxies. See text for reference on data sources.

tical. The best fit to all GCs gives an $\alpha=1.68 \pm 0.11$, where $\alpha$ is defined by the relation: $L \propto \sigma^{\alpha}$ (Djorgovski 1991). The only clear bias present in the correlation is the lack of low luminosity M31 clusters.

In summary, the correlation between $\mathrm{M}_{\mathrm{V}}$ and $\sigma$ of GCs shows that the structure of a GC is independent from galaxy environments: a spiral, a dwarf elliptical, or a magellanic galaxy. If confirmed to be an intrinsic characteristic of GCs by larger sample of clusters (from M31, for example), the $\mathrm{M}_{\mathrm{V}}-\sigma$ correlation can be one of the basis for answering the problem of the formation of globular clusters in galaxies.

\section{References}

Djorgovski S., 1991, ASP Conf. Ser., 13, 112

Dubath P., Mayor G., and Meylan G., 1993a, ASP Conf. Ser., 48, 557

Dubath P., Meylan G., and Mayor G., 1993b, $A p J, 400,510$

Harris W.E., 1991 ARAA, 29, 543

Peterson R.C., 1989, in Dynamics of Dense Stellar Systems, ed. D. Merrit, Cambridge University Press, p.161

Peterson R.C., 1993, ASP Conf. Ser., 48, 463

Peterson R.C., and Cudworth K.M., 1994, ApJ, 420, 612

Peterson R.C., Rees R.F., and Cudworth K.M., 1995, ApJ, 443, 124

Pryor C., and Meylan G., 1993, ASP Conf. Ser., 50, 357

Secker J., and Harris W.E., 1993, $A p J, 105,1358$

Stryker et al., 1985, ApJ, 367, 528

van den Bergh S., 1981, A\&A Sup. Ser., 46, 79

Zaggia S.R., Capaccioli M., Piotto G., 1995, $A \& A$, in preparation 